\title{
Direct Detection of penA gene Associated with Ceftriaxone-Resistant Neisseria gonorrhoeae FC428 Strain by Using PCR
}

\author{
David M. Whiley, Lebogang Mhango, \\ Amy V. Jennison, Graeme Nimmo, \\ Monica M. Lahra
}

The ceftriaxone-resistant Neisseria gonorrhoeae FC428 clone was first observed in Japan in 2015, and in 2017, it was documented in Denmark, Canada, and Australia. Here, we describe a PCR for direct detection of the penA gene associated with this strain that can be used to enhance surveillance activities.

$\mathrm{C}$ eftriaxone, either monotherapy or in dual therapy with azithromycin, is the mainstay of treatment of patients diagnosed with Neisseria gonorrhoeae infection in most settings (1). Therefore, the identification of any strains exhibiting resistance to ceftriaxone is of considerable public health concern. Until 2017, ceftriaxone-resistant strains of $N$. gonorrhoeae had been rare and typically sporadic, including in 2009, H041 in Japan (2); in 2010, F89 in France (3); in 2011, F89 in Spain (4); in 2013, A8806 in Australia (5); in 2014, GU140106 in Japan (6); and in 2015, FC428 and FC460 in Japan (7). However, there is now evidence of sustained international transmission of FC428, reported during 2017 in Canada (8) and Demark (9) (1 case each) and in Australia ( 2 cases) (10). Rapid and timely detection is pivotal to contain further spread of antimicrobial drugresistant $N$. gonorrhoeae. Here, we describe a real-time PCR protocol to facilitate enhanced surveillance for the FC428 clone. The study was approved by the University of Queensland Human Research Ethics Committee.

\section{The Study}

We designed a real-time PCR to target unique sequences on the penA gene of the FC428 N. gonorrhoeae clone (10).

Author affiliations: The University of Queensland, Brisbane, Queensland, Australia (D.M. Whiley, L. Mhango); Pathology Queensland Central Laboratory, Brisbane (D.M. Whiley, G. Nimmo); Queensland Health Forensic and Scientific Services, Archerfield, Queensland, Australia (A.V. Jennison); Griffith University, Gold Coast, Queensland, Australia (G. Nimmo); The Prince of Wales Hospital Randwich, Sydney, New South Wales, Australia (M.M Lahra); The University of New South Wales, Sydney (M.M. Lahra)

DOI: https://doi.org/10.3201/eid2408.180295
Modifications of penA, which encodes penicillin-binding protein 2 (PBP2), are a cause of cephalosporin resistance in $N$. gonorrhoeae. The FC428 clone harbors a mosaic penAallele, designated as PenA-60.001 by results of $N$. gonorrhoeae sequence typing for antimicrobial drug resistance (10), and encodes alterations including A $311 \mathrm{~V}$ and T483S that have previously been associated with $N$. gonorrhoeae ceftriaxone resistance in H041 (2) and A8806 $(2,5)$ strains. For this study, we designed 2 primers and 2 allele-specific probes (Table 1) to facilitate specific detection of the pen $A$ gene of FC428. In brief, the forward and reverse primers were designed to flank the $\mathrm{A} 311 \mathrm{~V}$ alteration; probe 1 was designed for detection of the $\mathrm{A} 311 \mathrm{~V}$ alteration; and probe 2 was designed to detect the wild-type A311 sequence. We added probe 2 to act as a blocker probe to limit binding of probe 1 with the wild-type sequence.

We prepared the reaction mix by using the QuantiTect Probe PCR Master Mix Kit (QIAGEN, Doncaster, Victoria, Australia). The reaction consisted of $12.5 \mu \mathrm{L}$ of the Master Mix, $10 \mathrm{pmol} / \mathrm{L}$ of forward and reverse primers (Table 1), $4.0 \mathrm{pmol} / \mathrm{L}$ of each probe, and $5.0 \mu \mathrm{L}$ of specimen nucleic acid, resulting in a total volume of $25 \mu \mathrm{L}$. We thermocycled the reaction mix by using the Rotor-Gene 6000 instrument (QIAGEN) and held it at $95^{\circ} \mathrm{C}$ for $15 \mathrm{~min}$, then cycled ( 45 cycles) at $95^{\circ} \mathrm{C}$ for $15 \mathrm{~s}$ and $60^{\circ} \mathrm{C}$ for $60 \mathrm{~s}$. We analyzed data by using the Rotor-Gene allelic discrimination software (QIAGEN).

We initially assessed the analytical performance of the assay by testing cultured isolates of $N$. gonorrhoeae $(\mathrm{n}=72)$ and commensal Neisseria and Moraxella species $(\mathrm{n}=111)$ (Table 2). We prepared these isolates by using a previously described heat-denaturation method (11). The $N$. gonorrhoeae isolates included the 2 FC428 strains recently documented in Australia (10), H041 (2) and A8806 (5); the ceftriaxoneresistant strains; and other local clinical $N$. gonorrhoeae isolates $(\mathrm{n}=68)$. Both $\mathrm{FC} 428$ isolates provided strong

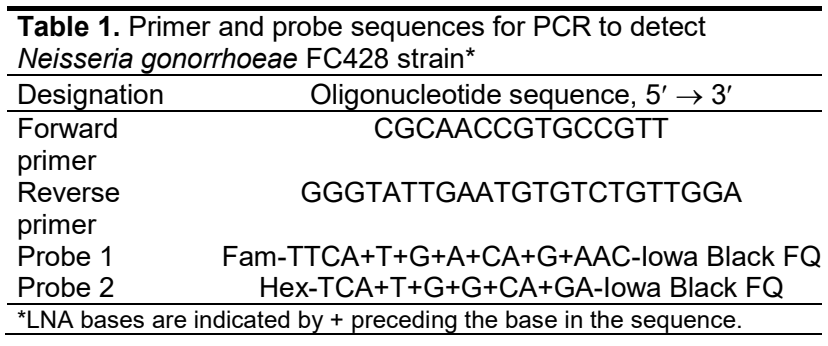


Table 2. Neisseria spp. isolates and specimens tested in development of PCR to detect Neisseria gonorrhoeae FC428 strain*

\begin{tabular}{|c|c|c|}
\hline \multirow[b]{2}{*}{ Isolates/samples } & \multicolumn{2}{|c|}{ PCR results for FC428 $\left(\mathrm{C}_{t}\right)$} \\
\hline & Probe 1 & Probe 2 \\
\hline \multicolumn{3}{|l|}{ Gonococcal species, $n=144$} \\
\hline \multicolumn{3}{|l|}{ Neisseria gonorrhoeae, $\mathrm{n}=72$} \\
\hline N. gonorrhoeae FC428, $\mathrm{n}=2(9) \dagger$ & Positive (19.8 and 18.17 cycles) & Negative \\
\hline N. gonorrhoeae H041, $\mathrm{n}=1(1)$ & $\begin{array}{l}\text { Negative } \\
\text { Cos }\end{array}$ & Negative \\
\hline N. gonorrhoeae A8806, $n=1$ (4) & Positive (37.8 cycles) & Negative \\
\hline N. gonorrhoeae, $\mathrm{n}=68 \ddagger$ & Negative & Negative \\
\hline \multicolumn{3}{|l|}{ Nongonococcal species, $\mathrm{n}=111$} \\
\hline N. cinerea, $n=4$ & Negative & Negative \\
\hline N. elongata, $\mathrm{n}=1$ & Negative & Negative \\
\hline N. flavescens, $\mathrm{n}=1$ & Negative & Negative \\
\hline N. lactamica, $\mathrm{n}=15$ & Negative & Negative \\
\hline N. lactamica, $\mathrm{n}=1$ & Positive (42.8 cycles) & Negative \\
\hline N. meningitidis, $\mathrm{n}=55$ & Negative & Negative \\
\hline N. meningitidis, $\mathrm{n}=1$ & Negative & Positive (32.6 cycles) \\
\hline N. mucosa, $\mathrm{n}=1$ & Negative & Negative \\
\hline N. polysacchareae, $n=4$ & Negative & Negative \\
\hline N. sicca, $\mathrm{n}=4$ & Negative & Negative \\
\hline N. subflava, $\mathrm{n}=14$ & Negative & Negative \\
\hline N. weaveri, $\mathrm{n}=1$ & Negative & Negative \\
\hline Moraxella catarrhalis, $\mathrm{n}=7$ & Negative & Negative \\
\hline M. osloensis, $\mathrm{n}=2$ & Negative & Negative \\
\hline \multicolumn{3}{|c|}{ N. gonorrhoeae NAAT-positive clinical specimens, $\mathrm{n}=358$} \\
\hline Urogenital, $n=172$ & Negative & Negative \\
\hline Anal swab, $n=81$ & Negative & Negative \\
\hline Throat swab, $n=95$ & Negative & Negative \\
\hline Other, $n=10$ & Negative & Negative \\
\hline
\end{tabular}

${ }^{*} \mathrm{C}_{\mathrm{t}}$, cycle threshold; NAAT, nucleic acid amplification test.

tIsolates A7846 and A7536.

fOther local clinical isolates collected in New South Wales, Australia.

positive signals by using probe 1 with cycle threshold $\left(\mathrm{C}_{\mathrm{t}}\right)$ values $<20$ cycles. The A8806 strain provided a late reaction at 37.8 cycles (probe 1), as did 2 commensal Neisseria strains: 1 N. lactamica isolate at 42.8 cycles for (probe 1 ), and $1 \mathrm{~N}$. meningitidis isolate at 32.6 cycles (probe 2). The Figure shows a sequence alignment of the partial penA sequences from these 3 isolates compared with the FC428 PenA-60.001 allele. The A8806 strain shows considerable sequence homology with PenA-60.001 (including 100\% match with the A311V Probe 1 sequence), albeit for 2 mutations in the forward primer designed to limit detection of A8806. We do not consider this a limitation of the assay because there has only been 1 reported case of infection with the A8806 strain. Neither the $N$. lactamica nor $N$. meningitidis isolates harbored the A311V alteration.

To compare detection limits, we tested 10-fold dilutions of FC428 DNA by both the FC428 PCR and a previously described in-house $N$. gonorrhoeae PCR, directed at the gonococcal porA and opa sequences (12). The in-house $N$. gonorrhoeae PCR had the lowest detection limit at 0.3 genome copies/reaction, whereas the detection limit of the FC428 PCR was 3.0 genome copies/reaction, indicating the FC428 PCR was $1 \log$ less sensitive than the diagnostic method.

We then applied the assay to a convenience panel of $N$. gonorrhoeae-positive clinical samples $(\mathrm{n}=358)$ submitted to Pathology Queensland Laboratory (Brisbane, Queensland, Australia) during February-September 2017 (Table 2). In brief, these samples comprised remnant nucleic acids from samples that tested positive for $N$. gonorrhoeae by the Cobas $4800 \mathrm{CT} / \mathrm{NG}$ test and were confirmed positive by using the in-house $N$. gonorrhoeae PCR (12). All samples provided negative results by the FC428 PCR, suggesting that the FC428 strain was not present in Queensland during this period.
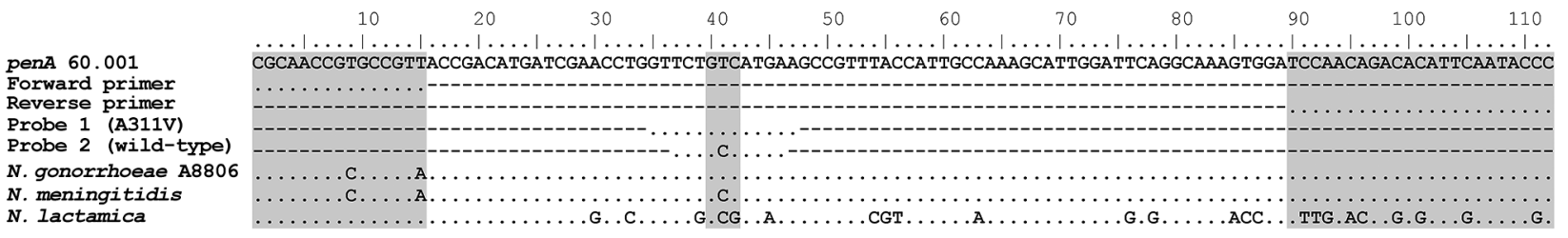

Figure. Sequence alignment showing the expected 112-bp PCR product for the PCR to detect Neisseria gonorrhoeae FC428 strain. PenA type 60.001 is provided as the reference sequence. Gray indicates the primer targets and the 311 codon within the probe target sequences. The penA sequences from the $N$. gonorrhoeae A8806, N. meningitidis, and $N$. lactamica isolates that cross-reacted with the FC428 PCR are also provided. Dots indicate sequence identity. 


\section{Conclusions}

Overall, our results suggest that the FC428 PCR is suitable for screening for the FC428 N. gonorrhoeae clone in clinical specimens for which culture is not available. The method could prove to be a strategic tool to enhance surveillance if FC428 continues to spread. We recommend that positive results be confirmed by, for example, DNA sequencing, particularly if the strain is detected in a pharyngeal sample in which other commensal Neisseria species are prevalent.

\section{Acknowledgments}

We thank Integrated DNA Technologies for technical support with oligonucleotide design, and the staff at the Molecular Diagnostic Unit of Pathology Queensland for their assistance with the study.

This study was funded by internal funds from the Australian Government Department of Health and Ageing. D.M.W. is a recipient of an NHMRC fellowship and receives research funding from SpeeDx Pty Ltd.

\section{About the Author}

Dr. Whiley is a principal research fellow at the Centre for Clinical Research, Faculty of Medicine, The University of Queensland, and a senior scientist at Pathology Queensland, Brisbane. His research interests include molecular diagnostics and antimicrobial resistance.

\section{References}

1. Unemo M, Jensen JS. Antimicrobial-resistant sexually transmitted infections: gonorrhoea and Mycoplasma genitalium. Nat Rev Urol. 2017;14:139-52. http://dx.doi.org/10.1038/nrurol.2016.268

2. Ohnishi M, Golparian D, Shimuta K, Saika T, Hoshina S, Iwasaku K, et al. Is Neisseria gonorrhoeae initiating a future era of untreatable gonorrhea?: detailed characterization of the first strain with high-level resistance to ceftriaxone. Antimicrob Agents Chemother. 2011;55:3538-45. http://dx.doi.org/10.1128/ AAC.00325-11

3. Unemo M, Golparian D, Nicholas R, Ohnishi M, Gallay A, Sednaoui P. High-level cefixime- and ceftriaxone-resistant Neisseria gonorrhoeae in France: novel penA mosaic allele in a successful international clone causes treatment failure. Antimicrob
Agents Chemother. 2012;56:1273-80. http://dx.doi.org/10.1128/ AAC.05760-11

4. Cámara J, Serra J, Ayats J, Bastida T, Carnicer-Pont D, Andreu A, et al. Molecular characterization of two high-level ceftriaxoneresistant Neisseria gonorrhoeae isolates detected in Catalonia, Spain. J Antimicrob Chemother. 2012;67:1858-60. http://dx.doi.org/ 10.1093/jac/dks162

5. Lahra MM, Ryder N, Whiley DM. A new multidrug-resistant strain of Neisseria gonorrhoeae in Australia. N Engl J Med. 2014;371:1850-1. http://dx.doi.org/10.1056/NEJMc1408109

6. Deguchi T, Yasuda M, Hatazaki K, Kameyama K, Horie K, Kato T, et al. New clinical strain of Neisseria gonorrhoeae with decreased susceptibility to ceftriaxone, Japan. Emerg Infect Dis. 2016;22:142-4. http://dx.doi.org/10.3201/eid2201.150868

7. Nakayama S, Shimuta K, Furubayashi K, Kawahata T, Unemo M, Ohnishi M. New ceftriaxone and multidrug-resistant Neisseria gonorrhoeae strain with a novel mosaic penA gene isolated in Japan. Antimicrob Agents Chemother. 2016;60:4339-41. http://dx.doi.org/10.1128/AAC.00504-16

8. Lefebvre B, Martin I, Demczuk W, Deshaies L, Michaud S, Labbe AC, et al. Ceftriaxone-resistant Neisseria gonorrhoeae, Canada, 2017. Emerg Infect Dis. 2018;24(2):381-383. https://dx.doi.org/10.3201/eid2402.171756

9. Terkelsen D, Tolstrup J, Hundahl Johnsen C, Lund O, Kiellberg Larsen H, Worning P, et al. Multidrug-resistant Neisseria gonorrhoeae infection with ceftriaxone resistance and intermediate resistance to azithromycin, Denmark, 2017. Euro Surveill. 2017;22(42):17.000659. https://dx.doi.org/ 10.2807/15607917.ES.2017.22.42.17-00659

10. Lahra M, Martin I, Demczuk W, Jennison AV, Lee K, Nakayama S, Lefebvre B, et al. Cooperative recognition of internationally disseminated ceftriaxone-resistant Neisseria gonorrhoeae strain. Emerg Infect Dis. 2018;24(4): 735-743. https://dx.doi.org/10.3201/eid2404.171873

11. Kugelman G, Tapsall JW, Goire N, Syrmis MW, Limnios A, Lambert SB, et al. Simple, rapid, and inexpensive detection of Neisseria gonorrhoeae resistance mechanisms using heatdenatured isolates and SYBR green-based real-time PCR. Antimicrob Agents Chemother. 2009;53:4211-6. http://dx.doi.org/ 10.1128/AAC.00385-09

12. Goire N, Nissen MD, LeCornec GM, Sloots TP, Whiley DM. A duplex Neisseria gonorrhoeae real-time polymerase chain reaction assay targeting the gonococcal porA pseudogene and multicopy opa genes. Diagn Microbiol Infect Dis. 2008;61:6-12. http://dx.doi.org/10.1016/j.diagmicrobio.2007.12.007

Address for correspondence: David M. Whiley, The University of Queensland, Faculty of Medicine, Centre for Clinical Research, UQCCR, Herston, Brisbane, Queensland 4029, Australia; email: d.whiley@uq.edu.au 